\title{
Unusual Local Recurrence with Distant Metastasis after Successful Endoscopic Submucosal Dissection for Colorectal Mucosal Cancer
}

\author{
Hyo Jeong Lee ${ }^{1}$, Byong Duk Ye ${ }^{2}$, Jeong-Sik Byeon ${ }^{2}$, Jihun Kim ${ }^{3}$, Young Soo Park ${ }^{3}$, Yong Sang Hong ${ }^{4}$, Yong Sik Yoon \\ and Dong-Hoon Yang ${ }^{2}$
}

${ }^{1}$ Health Screening and Promotion Center, Departments of ${ }^{2}$ Gastroenterology, ${ }^{3}$ Pathology, ${ }^{4}$ Oncology, and ${ }^{5}$ Colon and Rectal Surgery, Asan Medical Center, University of Ulsan College of Medicine, Seoul, Korea

Intramucosal colorectal cancer (CRC) is thought not to metastasize because the colonic lamina propria lacks lymphatics. Only a few recent case reports have suggested lymph node metastasis from intramucosal CRC, but there is no clear evidence supporting the metastatic potential of intramucosal CRC. Hence, endoscopic resection is regarded as curative treatment for intramucosal CRC. This report describes two cases of unusual local recurrence with distant metastasis in patients who had previously undergone successful endoscopic submucosal dissection for intramucosal CRC. The recurrent colorectal lesions developed at the site of the previous endoscopic submucosal dissection scars in a relatively short-term period, and the pathologic findings showed an "undermining" invasion pattern without surrounding mucosal change. Based on the clinical course and pathological findings, we concluded that the second colorectal lesions were recurrences rather than de novo cancers. Clin Endosc 2017;50:91-95

Key Words: Colorectal neoplasms; Intramucosal carcinoma; Endoscopic submucosal dissection; Recurrence; Neoplasm metastasis

\section{INTRODUCTION}

Colorectal cancer (CRC) with invasion limited to the lamina propria (LP) is defined as intramucosal carcinoma. The current consensus is that intramucosal CRC should not metastasize because colonic LP lacks lymphatics. ${ }^{1}$ Hence, intramucosal CRC is classified as "Tis" in the tumor, node, metastasis (TNM) staging system, and endoscopic resection is regarded as adequate treatment. ${ }^{2,3}$ However, recent reports have described local recurrence with distant metastasis after surgical resection for poorly differentiated intramucosal rectal cancer. ${ }^{4}$ Data regarding metastasis in intramucosal tumors are still lacking, and the metastatic potential of intramucosal CRC

Received: March 24, 2016 Revised: August 2, 2016

Accepted: August 3, 2016

Correspondence: Dong-Hoon Yang

Department of Gastroenterology, Asan Medical Center, University of Ulsan College of Medicine, 88 Olympic-ro 43-gil, Songpa-gu, Seoul 05505, Korea

Tel: +82-2-3010-5809, Fax: +82-2-3010-6157, E-mail: dhyang@amc.seoul.kr

(cc) This is an Open Access article distributed under the terms of the Creative Commons Attribution Non-Commercial License (http://creativecommons.org/ licenses/by-nc/3.0) which permits unrestricted non-commercial use, distribution, and reproduction in any medium, provided the original work is properly cited. remains unclear.

Herein, we report two rare cases of local recurrence with distant metastasis in patients who previously underwent endoscopic submucosal dissection (ESD) for intramucosal CRCs.

\section{CASE REPORTS}

\section{Case 1}

A 67-year-old woman underwent ESD for a 5.6-cm mixednodular type laterally spreading tumor (LST) in the rectum (Fig. 1A, B). Neither a non-lifting sign nor significant submucosal fibrosis was identified. The specimen was fixed in $10 \%$ formalin, paraffin-embedded, and evaluated after being cut into 2-mm-thick slices. The histology showed a well-differentiated adenocarcinoma confined to the LP without lymphovascular invasion and with clear resection margins (Fig. 1C). Abdomino-pelvic computed tomography (CT) did not show any lymph node or distant metastasis. Follow-up sigmoidoscopy at 8 months showed only a scar (Fig. 1D). However, she was admitted with sacral area pain at 17 months after ESD. 

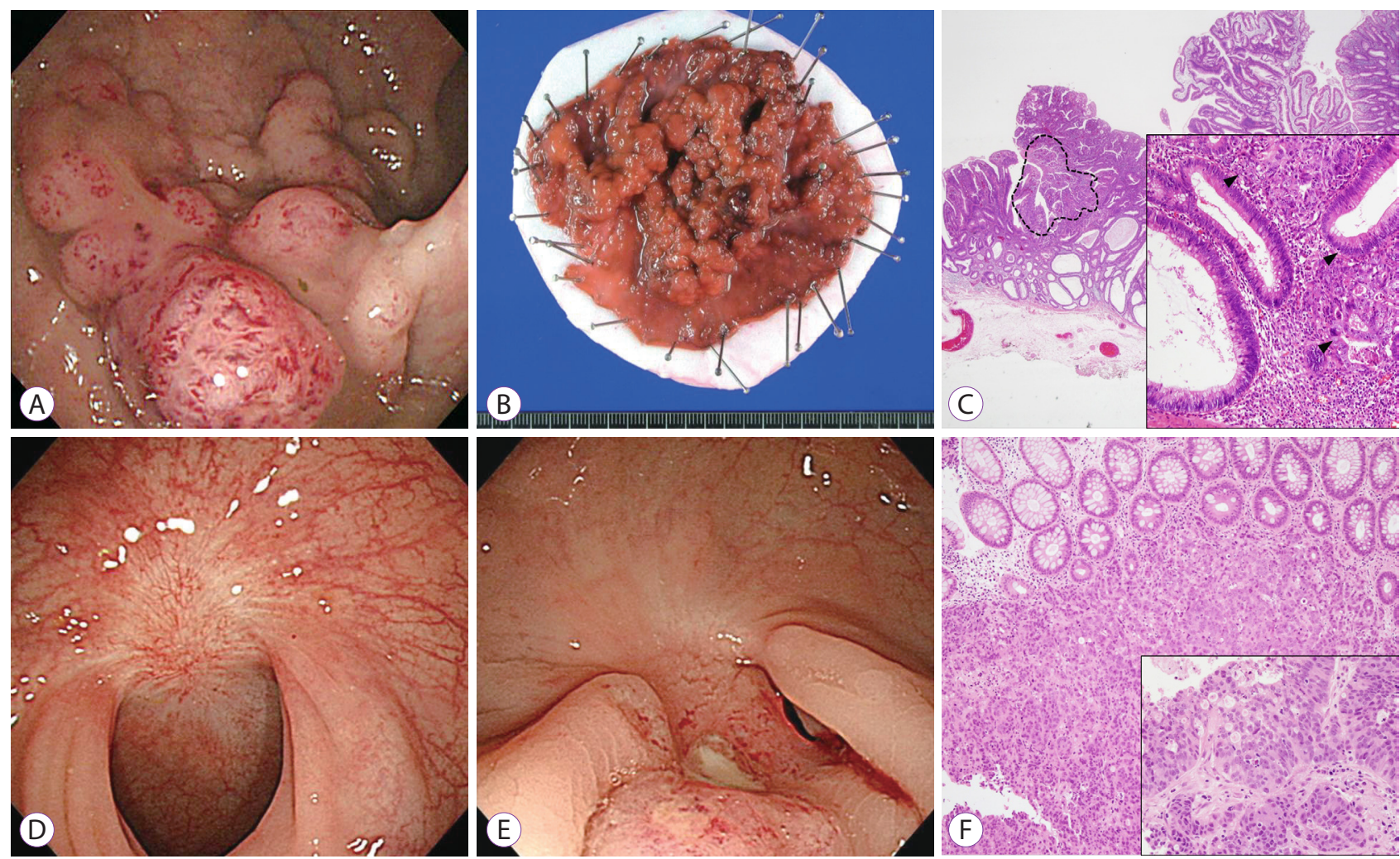

Fig. 1. (A) Colonoscopic finding showing a mixed-nodular type laterally spreading tumor measuring $5.6 \mathrm{~cm}$ in diameter. (B) Gross endoscopic submucosal dissection (ESD) specimen of the primary lesion. (C) Pathological findings for the ESD specimen showing multiple foci of adenocarcinoma component in the bulky laterally spreading adenoma. The least-differentiated component is highlighted by a dashed line (H\&E stain, $\times 10$ ). Higher magnification of the least-differentiated area, showing solid and cribriform architecture and multiple foci of the invasive front, consisting of small, infiltrative tumor glands (arrowheads) (inset: H\&E stain, $\times 200$ ). (D) Follow-up sigmoidoscopy in 8 months shows a scar. (E) Sigmoidoscopy performed 17 months after ESD, showing mucosal elevation with central ulceration at the previous procedure site, which is suggestive of extrinsic infiltrative cancer. (F) Endoscopic biopsy obtained from the previous ESD site, showing a poorly differentiated adenocarcinoma sitting under normal colonic crypts (H\&E stain, $\times 100)$. Needle biopsy specimen of a pulmonary metastasis showing similar morphology of tumor glands to that of the previous ESD specimen in terms of solid and cribriform architecture (inset: H\&E stain, $\times 200$ ).

Sigmoidoscopy showed an extrinsic infiltrative lesion at the previous ESD site (Fig. 1E). CT showed a perirectal mass, enlarged perirectal lymph nodes, and multiple lung nodules. The pathological findings of the previous ESD site lesion revealed a poorly differentiated adenocarcinoma under normal colonic crypts (Fig. 1F). This undermining invasion pattern without surrounding mucosal change suggested that the recurrent lesion represented in situ recurrence rather than de novo cancer. The histology of lung nodules (Fig. 1F, inset) showed a poorly differentiated adenocarcinoma that was positive for cytokeratin 20 and negative for cytokeratin 7 on immunohistochemistry, suggesting pulmonary metastasis of enteric type adenocarcinoma. Following these findings, the entire original ESD specimen was re-examined after slicing the paraffin-embedded blocks to check for the presence of missed submucosal invasive foci smaller than $2 \mathrm{~mm}$, the routine slice thickness for histologic review of an ESD specimen at our center. ${ }^{5}$ At low magnification, the bulky, laterally spreading adenoma contained multiple foci of adenocarcinoma component (Fig. 1C). Higher magnification of the least differentiated area showed solid and cribriform architecture and multiple foci of the invasive front, consisting of small, infiltrative tumor glands (Fig. 1C, inset). However, neither submucosal invasion nor lymphovascular invasion was identified, even with CD34 and D2-40 immunostaining.

\section{Case 2}

A 62-year-old woman underwent en bloc ESD for a 6-cm, mixed-nodular LST in the ascending colon (Fig. 2A, B). No submucosal fibrosis was identified during ESD. The histological examination showed a laterally spreading adenoma with a small fraction of moderately differentiated adenocarcinoma component (Fig. 2C, dashed line). At higher magnification, the invasive tumor cells formed large, irregularly shaped tubules and had occasional goblet cells, and the surrounding stroma was desmoplastic (Fig. 2C, inset). The adenocarcinoma component was confined to the LP without lymphovascular invasion. As expected in the endoscopic findings (Fig. 2B, arrowheads), the resection margins showed severe cautery artifact and low-grade dysplasia involved the resection margins. 

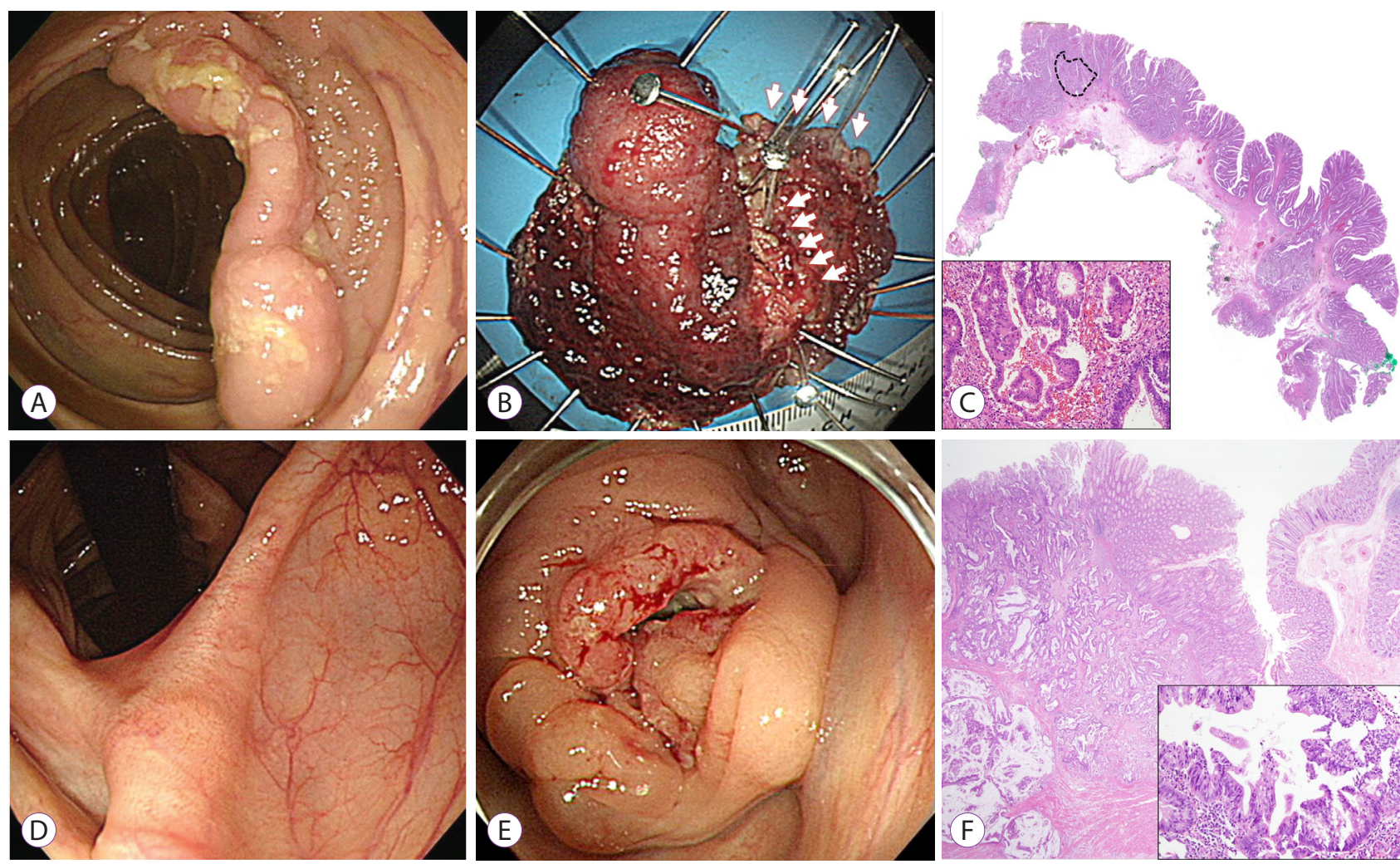

Fig. 2. (A) Colonoscopic finding showing a mixed-nodular type laterally spreading tumor measuring $6.0 \mathrm{~cm}$ in diameter. (B) Severe cauterization at the margin and the middle of the tumor is suspected in the endoscopic image (arrows). (C) Pathological findings for the endoscopic submucosal dissection (ESD) specimen showing a laterally spreading adenoma with a frankly invasive adenocarcinoma component (dashed line, H\&E stain, slide scan without magnification). At higher magnification, invasive tumor cells form large, irregularly shaped tubules and have occasional goblet cells. The surrounding stroma is desmoplastic (inset: H\&E stain, $\times 200)$. (D) Follow-up colonoscopy at 12 months shows only a scar. (E) Colonoscopy at 34 months after ESD shows an ulcerofungating mass that encircles the lumen at the previous ESD site. (F) Pathological findings of resected tumor show an "undermining" invasion pattern without surrounding mucosal change (H\&E stain, $\times 10)$. The cytomorphology of the resected tumor is similar to that of the invasive component of the previous ESD specimen (inset: H\&E stain, $\times 200$ ).

Abdomino-pelvic CT did not show any lymph node or distant metastasis. Follow-up colonoscopy at 12 months showed only a scar (Fig. 2D).

However, the patient was admitted with abdominal pain at 34 months after ESD. Colonoscopy showed an ulcerofungating mass encircling the lumen at the previous ESD site (Fig. 2E). Positron emission tomography showed a 1.1-cm hypermetabolic mass in the liver. She underwent right hemicolectomy with hepatic segmentectomy. The colectomy specimen showed a large subserosal tumor with atypical ulcer, which was characterized by the absence of surrounding hyperplastic mucosa and multiple foci of intervening non-neoplastic mucosal islands. Microscopically, the colectomy specimen showed an "undermining" invasion pattern, in which total tumor volume was disproportionately larger than that expected from the size of the mucosal lesion. Furthermore, surrounding mucosa and mucosal "islands" in the ulcer base did not show any preneoplastic changes (Fig. 2F). At higher magnification, the cytomorphology of the resected tumor was similar to that of the invasive component of the previous ESD specimen (Fig.
$2 \mathrm{~F}$, inset). The pathological findings of the hepatic segmentectomy specimen showed findings similar to those of the colon specimen, suggesting hepatic metastasis from the colon cancer. Similar to case 1, the entire ESD specimen was reviewed again with additional sections of the paraffin-embedded blocks and with CD34 and D2-40 immunostaining. However, submucosal invasive foci and lymphovascular invasion were not identified.

We concluded this case was a recurrence of colon cancer with hepatic metastasis in a patient who previously underwent ESD for intramucosal colon cancer, because the second colon lesion developed precisely at the previous ESD scar site during a 34-month follow-up period, and the pathologic finding showed an "undermining" invasion pattern without surrounding preneoplastic mucosal change.

\section{DISCUSSION}

Generally, it is thought that intramucosal CRC does not 


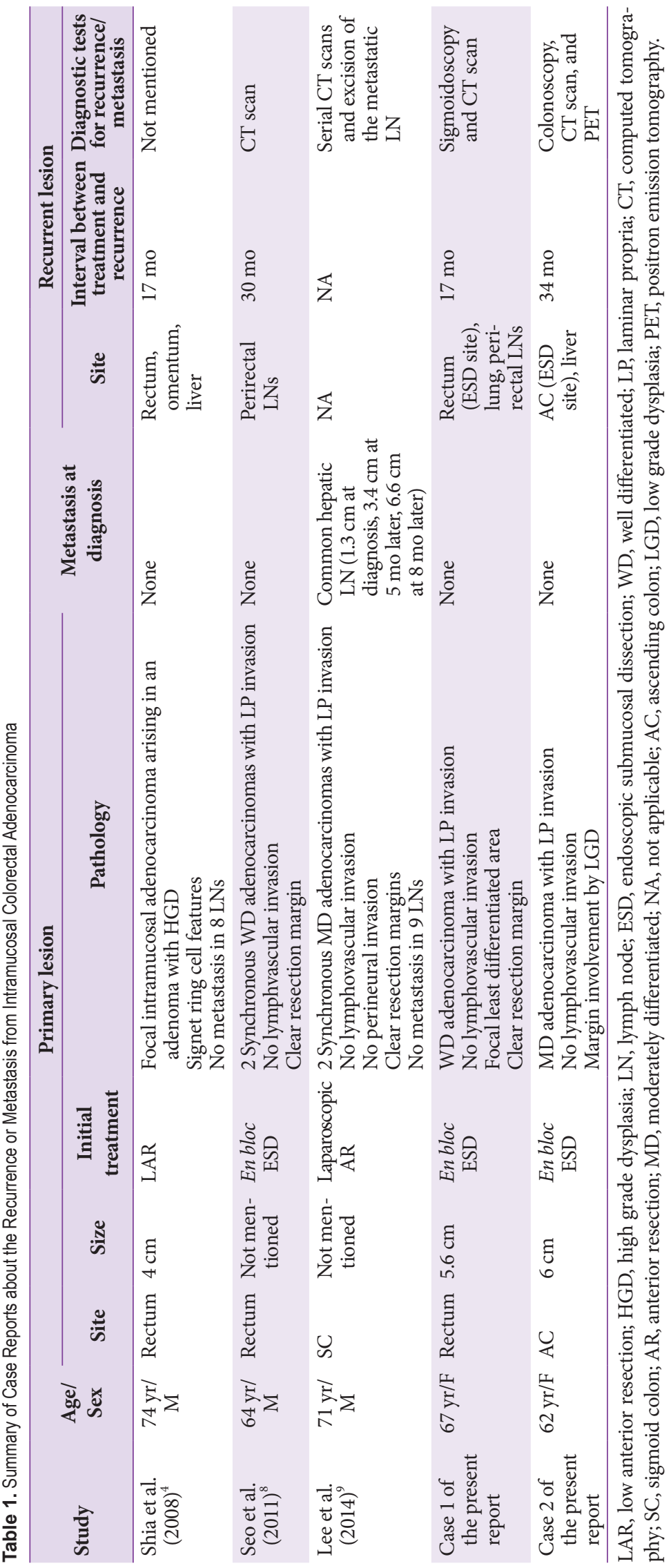

carry the risk of metastasis, ${ }^{2,3}$ and thus, endoscopic procedures such as ESD or endoscopic mucosal resection, are accepted as curative modalities for intramucosal CRC. ${ }^{2,3,67}$ However, recent studies have reported several instances of metastasis from intramucosal CRC. ${ }^{4,89}$ Nation-based survival data for the revised staging system in the seventh edition of the American Joint Committee on Cancer also showed that a small proportion of Tis colon cancers have lymph node metastasis. ${ }^{10}$ Table 1 shows the characteristics of intramucosal CRCs with metastasis at diagnosis or recurrent intramucosal CRCs during follow-up in previous studies. Interestingly, two of five cases had two synchronous intramucosal CRCs as primary lesions, ${ }^{8,9}$ and the other three cases had huge intramucosal CRCs as primary lesions. ${ }^{4}$ One previously reported case had focal signet ring cell features, ${ }^{4}$ and case 1 in the present report showed a focal least-differentiated area, although the metastatic potential of these focal poorly differentiated features is uncertain. Therefore, metastasis from intramucosal colorectal adenocarcinoma may be theoretically impossible, but may not be a never-happened event.

We present two cases with unusual features of local recurrence with distant metastasis in patients who previously underwent en bloc ESD for intramucosal CRC. We initially considered the possibility of de novo cancer. However, in these cases, second lesions developed precisely at the ESD scar sites in a relatively short-term period. In addition, an undermining invasion pattern without overlying mucosal change strongly suggested recurrence of the previous intramucosal CRCs. The second lesions in both cases showed pathological findings similar to the least-differentiated component of previous ESD specimens. Moreover, the cancers at the previous ESD sites showed an "undermining" invasion pattern, in which most tumor cells were observed underneath non-neoplastic mucosa without any preneoplastic changes. These appearances are extremely rare in de novo cancer.

The mechanism of recurrence as advanced or metastatic carcinoma after ESD is unclear. Although a previous report suggested that focal lymphatic tumor invasion at the base of the mucosa might be a possible route for metastasis in intramucosal CRC, ${ }^{4}$ no lymphatic tumor invasion was identified in our present cases. According to a meta-analysis, intraoperative rectal washout can 
reduce local recurrence after rectal cancer surgery. ${ }^{11}$ Moreover, a recent study revealed that tumor cells can be exfoliated into the intestinal lumen during colorectal ESD, ${ }^{12}$ although little is known about the clinical significance of exfoliated tumor cells related to endoscopic procedures. Interestingly, a case report suggested that colorectal adenocarcinoma cells might be implanted into the artificial ulcer after endoscopic resection. ${ }^{13}$ As ESD for our primary lesions needed prolonged time, neoplastic cells shed from the tumor surface during the procedure might be implanted on the exposed submucosal layer or directly into the damaged lymphatics of the artificial ulcer. In case 2, which showed lateral margin involvement by adenoma and severe cautery artifact, the remaining neoplastic cells at the margins might be the source of recurrence, although the progression to invasive cancer was extraordinarily fast. The presence of missed unfavorable histologic findings such as focal deep submucosal invasive cancer, even after meticulous histologic reexamination, might be another mechanism of recurrence. The presence of an extremely rare, but still unknown subtype of intramucosal CRC cannot be excluded.

In conclusion, we report two rare cases of local recurrence with distant metastasis in patients who previously underwent successful ESD for intramucosal CRC. Regardless of the mechanism, the rare possibility of recurrence may be considered during surveillance after en bloc ESD of huge intramucosal CRCs. The appropriate surveillance interval after ESD of huge intramucosal CRCs is still uncertain, as most postpolypectomy surveillance guidelines are based on the data retrieved from non-ESD-related studies. ${ }^{14-16}$ Given that both local and distant recurrences were identified at 17, 30, and 34 months after ESD in our cases and in previous reports, ${ }^{4,8}$ yearly surveillance for 3 years with colonoscopy and/or CT might be acceptable if a rare but possible recurrence is a concern after ESD of huge intramucosal CRCs. However, additional data should be accumulated to suggest a reasonable surveillance strategy after endoscopic treatment of huge intramucosal CRCs.

\section{Conflicts of Interest}

The authors have no financial conflicts of interest.

\section{REFERENCES}

1. Fenoglio CM, Kaye GI, Lane N. Distribution of human colonic lymphatics in normal, hyperplastic, and adenomatous tissue. Its relationship to metastasis from small carcinomas in pedunculated adenomas, with two case reports. Gastroenterology 1973;64:51-66.

2. Engstrom PF, Arnoletti JP, Benson AB 3rd, et al. NCCN clinical practice guidelines in oncology: colon cancer. J Natl Compr Canc Netw 2009;7:778-831.

3. Engstrom PF, Arnoletti JP, Benson AB 3rd, et al. NCCN clinical practice guidelines in oncology: rectal cancer. J Natl Compr Canc Netw 2009;7:838-881.

4. Shia J, Klimstra DS. Intramucosal poorly differentiated colorectal carcinoma: can it be managed conservatively? Am J Surg Pathol 2008;32:15861588 .

5. Bae JH, Yang DH, Lee JY, et al. Clinical outcomes of endoscopic submucosal dissection for large colorectal neoplasms: a comparison of protruding and laterally spreading tumors. Surg Endosc 2016;30:1619-1628.

6. Nivatvongs S. Surgical management of early colorectal cancer. World J Surg 2000;24:1052-1055.

7. Saito Y, Uraoka T, Matsuda T, et al. Endoscopic treatment of large superficial colorectal tumors: a case series of 200 endoscopic submucosal dissections (with video). Gastrointest Endosc 2007;66:966-973.

8. Seo HJ, Kim YJ, Cho KB, et al. Nodal metastasis after successful endoscopic submucosal dissection for colorectal mucosal cancer. Endoscopy 2011;43 Suppl 2 UCTN:E374-E375.

9. Lee KH, Kim JS, Cheon KS, Song IS, Kang DY, Kim JY. TisNOM1 sigmoid colon cancer: a case report. Ann Coloproctol 2014;30:141-146.

10. Gunderson LL, Jessup JM, Sargent DJ, Greene FL, Stewart AK. Revised $\mathrm{TN}$ categorization for colon cancer based on national survival outcomes data. J Clin Oncol 2010;28:264-271.

11. Matsuda A, Kishi T, Musso G, et al. The effect of intraoperative rectal washout on local recurrence after rectal cancer surgery: a meta-analysis. Ann Surg Oncol 2013;20:856-863.

12. Inoue $\mathrm{T}$, Fujii $\mathrm{H}$, Koyama $\mathrm{F}$, et al. Intraluminal lavage to remove exfoliated tumor cells after colorectal endoscopic submucosal dissection. Surg Endosc 2016;30:2773-2778.

13. Tajika M, Niwa Y, Bhatia V, et al. A first report of tumor cell implantation after EMR in a patient with rectosigmoid cancer. Gastrointest Endosc 2012;75:1117-1118.

14. Yang $\mathrm{DH}$, Hong $\mathrm{SN}$, Kim $\mathrm{YH}$, et al. Korean guidelines for postpolypectomy colonoscopy surveillance. Clin Endosc 2012;45:44-61.

15. Lieberman DA, Rex DK, Winawer SJ, et al. Guidelines for colonoscopy surveillance after screening and polypectomy: a consensus update by the US multi-society task force on colorectal cancer. Gastroenterology 2012;143:844-857.

16. Hassan C, Quintero E, Dumonceau JM, et al. Post-polypectomy colonoscopy surveillance: European society of gastrointestinal endoscopy (ESGE) guideline. Endoscopy 2013;45:842-851. 